Projets

de paysage

\section{Projets de paysage}

Revue scientifique sur la conception et l'aménagement de l'espace

$22 \mid 2020$

Forêt et paysage

\title{
Géohistoire du massif forestier d'Écouves (Orne, Normandie)
}

Premiers résultats de recherche fondés sur les informations biogéographiques et archéologiques issues des sources historiques A Geohistory of the Écouves Forest Massif (Orne, Normandy) - Initial Research Findings Based on Biogeographic and Archaeological Information from Historical Sources

Nicolas Blanchard, Damase Mouralis et Dominique Todisco

\section{OpenEdition}

\section{Journals}

\section{Édition électronique}

URL : http://journals.openedition.org/paysage/8113

DOI : 10.4000/paysage.8113

ISSN : 1969-6124

\section{Éditeur :}

École nationale supérieure du paysage de Versailles-Marseille, Institut national des sciences appliquées Centre Val de Loire - École de la nature et du paysage, École nationale supérieure d'architecture et de paysage de Bordeaux, École nationale supérieure d'architecture et de paysage de Lille, Agrocampus Angers

Référence électronique

Nicolas Blanchard, Damase Mouralis et Dominique Todisco, « Géohistoire du massif forestier d'Écouves (Orne, Normandie) », Projets de paysage [En ligne], 22 | 2020, mis en ligne le 21 juillet 2020, consulté le 24 juillet 2020. URL : http://journals.openedition.org/paysage/8113 ; DOI : https://doi.org/ $10.4000 /$ paysage.8113

Ce document a été généré automatiquement le 24 juillet 2020

Projets de paysage 


\section{Géohistoire du massif forestier d'Écouves (Orne, Normandie)}

Premiers résultats de recherche fondés sur les informations biogéographiques et archéologiques issues des sources historiques

A Geohistory of the Écouves Forest Massif(Orne, Normandy) - Initial Research Findings Based on Biogeographic and Archaeological Information from

Historical Sources

Nicolas Blanchard, Damase Mouralis et Dominique Todisco

1 L'arrivée de nouvelles technologies dans l'étude des forêts, notamment la télédétection et l'imagerie Light Detection And Ranging (Lidar), a remis en cause certains concepts selon lesquels les espaces forestiers de l'Ouest européen, actuellement en place, correspondraient à des reliquats historiques de l'époque antique ou médiévale (Musset, 1961 ; Louise, 1988). De fait, ces technologies ont invité à reconsidérer ou à nuancer les notions de forêts anciennes des régions tempérées et plus spécifiquement des grands massifs Français. Murielle Georges-Leroy et al. (2009) ont ainsi montré, grâce à l'imagerie Lidar, que la forêt de Haye (Meurthe-et-Moselle) s'est étendue au détriment d'anciens agrosystèmes antiques. De fait, l'objectif de la présente étude se situe dans ce contexte scientifique qui vise à documenter : 1) la dynamique d'anthropisation des terroirs actuellement sous couvert forestier et 2), de comprendre l'influence de cette anthropisation (ante-forestière) dans les processus d'évolution (apparition, fixation, altération) du paysage forestier. Les forêts françaises, comme palimpseste spatial, conservent ainsi des traces d'anthropisations antérieures à l'état boisé actuel, liées aux pratiques sylvicoles ou agraires anciennes.

2 Afin d'établir une première chronologie des trajectoires évolutives de la forêt d'Écouves, nous croisons l'ensemble des sources disponibles (archives historiques, terrain, observations botaniques) dans le but d'appréhender l'évolution des faits anthropiques et leurs dynamiques spatiales. Dans la mesure où l'information scientifique reste relativement limitée pour le territoire à l'étude, la mise au jour de quelques structures agraires à partir du dépouillement des archives a motivé une 
mobilisation exhaustive de sources historiques ( $\mathrm{xII}^{\mathrm{e}}-\mathrm{xx}^{\mathrm{e}}$ siècle). Cette démarche intervient également en amont d'un vol Lidar effectué sur la forêt d'Écouves en février 2019 entrepris par la direction régionale des Affaires culturelle (Drac) et de l'office national des forêts (ONF). Si des études similaires ont vu le jour, plus rares sont celles qui ont dépassé la seule exploitation de la carte ancienne. Dans le but de documenter à la fois la dynamique d'évolution de la végétation sur la longue durée (diachronie) et l'influence des pratiques anthropiques anciennes sur le paysage actuellement occupé par la forêt d'Écouves, les sources historiques ont donc constitué le corpus principal de cette étude. Composée essentiellement des documents d'aménagement de la forêt d'Écouves $(1667,1785,1861)$, cette ressource documentaire a été complétée par une prospection archéologique dans la partie domaniale du massif où des anomalies topographiques et botaniques ont été mises en évidence. Ainsi, l'étude du fonds d'archives conservé aux Archives départementales de l'Orne (ADO) ainsi qu'à la Bibliothèque nationale de France (BNF) a permis d'illustrer l'évolution du massif forestier sur cinq siècles, offrant la possibilité d'entreprendre une démarche géohistorique par l'analyse et la compréhension de la construction de l'espace (dynamiques paysagères, transmission des formes) sur la longue durée (approche diachronique).

3 Ainsi, l'exemple de la forêt d'Écouves illustre la diversité des informations apportées par les archives historiques dans la reconstitution des paysages anciens et permet de préciser les types d'informations qu'il est possible d'en extraire. En outre, notre étude permet de souligner les spécificités géographiques du massif étudié. En effet, nous partons de l'hypothèse selon laquelle la forêt d'Écouves diffère des forêts de plaines et de plateaux étudiées jusqu'alors dans le quart nord-ouest de la France, notamment du fait de sa topographie (pentes et expositions) caractérisée par des reliefs importants (414 $\mathrm{m}$ d'altitude) et des spécificités pédologiques (acidité des sols) liées en partie à l'altération de la roche mère (grès armoricain). Ce déterminisme semble à première vue avoir restreint les activités agropastorales aux seules lisières forestières actuelles.

4 Cette étude resitue dans une première partie la forêt d'Écouves dans son contexte scientifique et ses spécificités géographiques. La seconde partie présente les méthodes et outils utilisés en soulignant l'apport du croisement des sources historiques avec la prospection pédestre et les observations botaniques. Une dernière partie expose les premiers résultats montrant la diversité des formes d'anthropisation préforestières ainsi que les dynamiques d'évolution du paysage forestier.

\section{Contexte géohistorique de la forêt d'Écouves}

\section{La forêt domaniale d'Écouves}

\section{Une forêt collinéenne dans un pays de plaine}

Le massif forestier d'Écouves est situé dans l'Orne (Normandie) et coiffe les derniers contreforts orientaux du Massif armoricain, à la limite du Bassin parisien. Cette situation s'illustre par la présence de reliefs marqués dont le point culminant de la Normandie (414 m d'altitude). Le massif se développe principalement sur les assises géologiques précambriennes et primaires à l'origine de sols relativement pauvres en éléments nutritifs. Ces derniers sont donc majoritairement acides, sous forme de podzols et présentent un déficit en calcium en relation avec une roche mère 
principalement composée de grès armoricain de May (ONF, 2004). La grande majorité des stations forestières est associée à des sols acides à hyperacides, liés à des engorgements d'eau ou à d'importants affleurements de la roche mère. Seules les lisières de l'adret ainsi qu'un grand quart sud-est - reposant sur des schistes correspondent à des stations faiblement à moyennement acides. Avec ses presque 15000 ha, le massif forestier, composé d'environ 8000 ha de forêt domaniale et de 7000 ha de forêts privées, correspond actuellement à l'un des plus vastes ensembles forestiers de la Normandie.

Figure 1. Localisation de la zone étudiée

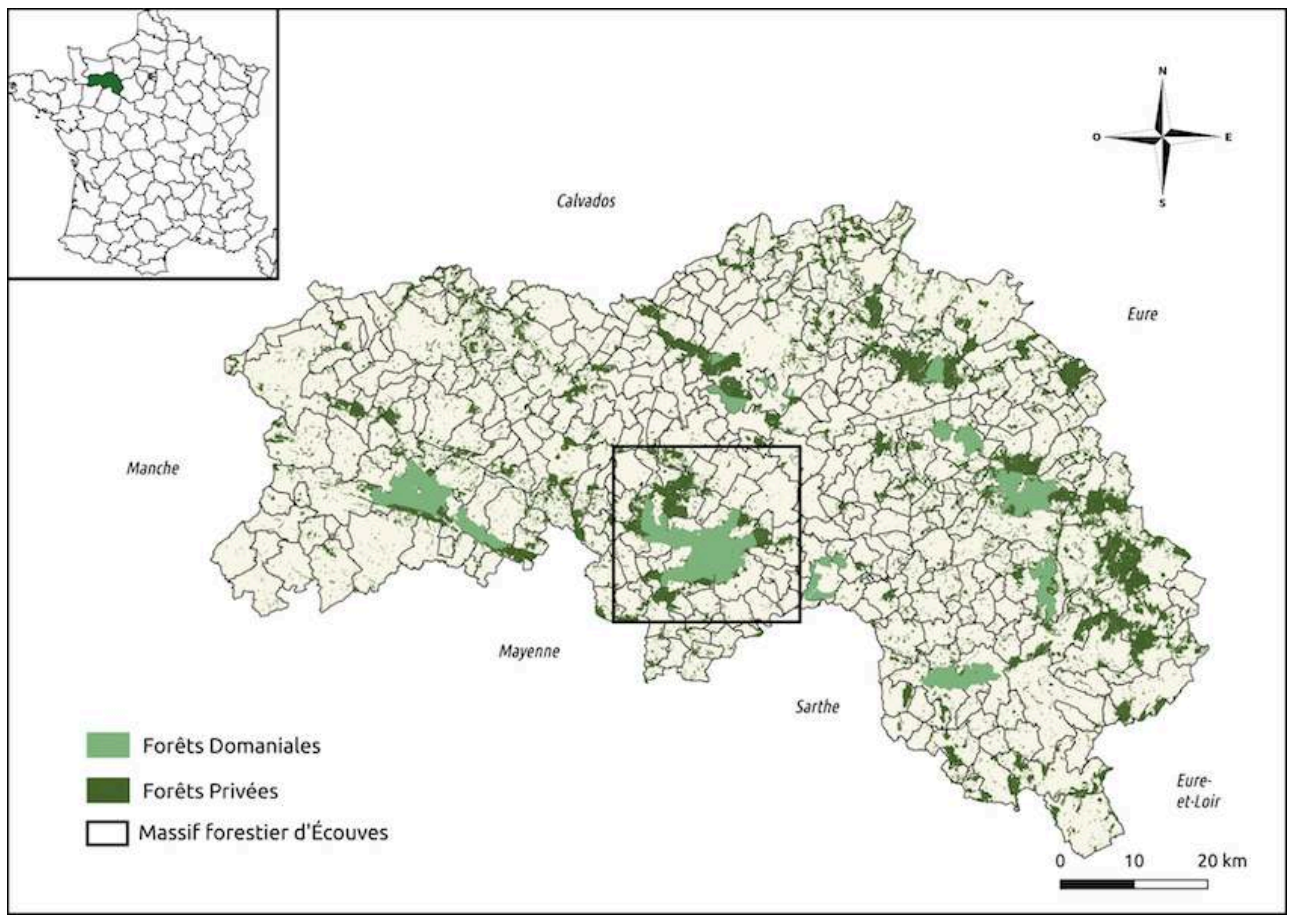

Sources : IGN/ONF/Nicolas Blanchard.

\section{Végétation actuelle du massif}

Le peuplement forestier et, plus largement, la végétation de la forêt domaniale d'Écouves sont très hétérogènes (Piriou et al., 2011 et 2013) même si celle-ci se caractérise par certains «traits de caractères" phytosociologiques en raison de la nature du sol. À ce titre, Bernard Jabiol (1985) indique qu'en Écouves, l'acidité affecte tous les matériaux rencontrés avec un $\mathrm{pH}$ des sols généralement inférieur à 5 . De fait, la végétation présente est principalement acidiphile, incluant la fougère aigle (Pteridium aquilinum), la callune (Calluna vulgaris) ou encore le myrtillier (Vaccinium myrtillus). Le peuplement forestier est composé à $48 \%$ de feuillus et à $47 \%$ de résineux, les $5 \%$ restants sont constitués de vides boisables et de clairières (ONF, 2004). Le chêne sessile ( $24 \%)$ et le hêtre $(22 \%)$ dominent les feuillus tandis que les peuplements de résineux sont davantage hétérogènes. Introduits dans le massif à partir des années 1820-1830, le sapin pectiné (14\%), le pin sylvestre (7\%), l'épicéa (11\%) et le douglas $(9 \%)$ occupent les milieux tourbeux, paratourbeux comme les lignes de crêtes. 


\section{Historique général de la forêt}

7 Du point de vue historique, les premières mentions de la forêt d'Écouves apparaissent dès les XII $^{\mathrm{e}}$-XIII ${ }^{\mathrm{e}}$ siècles, sous la forme de nemore scoparum ${ }^{1}$, du latin nemus qui désigne une forêt renfermant des pâtures (Wirth-Jaillard, 2019). À l'origine propriété des comtes d'Alençon, la forêt est entourée de propriétés vassaliques (bois Mallet, bois de Roche Elie, haie de la Ferrière) et des bois appartenant à des congrégations religieuses comme les bois de Mesnilgault, dépendant de l'abbaye de Saint-Martin de Sées, ou encore la forêt de Bois-l'Évêque. La forêt d'Écouves devient forêt royale au début du XIII ${ }^{\mathrm{e}}$ siècle. Entre cette période et l'époque moderne, peu de sources permettent de documenter son histoire, en raison notamment de la disparition de certains documents ${ }^{2}$. En 1667, elle est couronnée de 350 bornes de granite, matérialisant les limites du domaine royal. En 1774, elle devient la propriété du comte de Provence qui réorganise l'aménagement forestier. Ce dernier fait ouvrir de nombreuses routes pour faciliter la vidange des bois et fait placer l'aménagement des peuplements forestiers à $96 \%$ en taillis. Passé 1800, les peuplements forestiers sont fortement dégradés par les activités des forges et des verreries, dont les établissements, nombreux dans la région, étaient les principaux destinataires de l'exploitation forestière. Afin de repeupler la forêt, on y introduit à partir des années 1820-1830 de nouvelles essences comme le pin sylvestre et l'épicéa. Elle est durement touchée par la tempête Lothar en décembre 1999. L'aménagement forestier actuel (2004-2023) touche à sa fin et a amené l'ONF à effectuer un vol Lidar pour anticiper le futur document d'aménagement et l'intégration du patrimoine archéologique dans la gestion forestière.

\section{État de l'art}

\section{Les données archéologiques}

8 D'un point de vue général, la carte archéologique des 21 communes sur lesquelles s'étend l'actuel massif d'Écouves ne recense que 47 notices dont 9 en contexte forestier (structures, voies et dépôts), et reste documentée par une littérature datant principalement du XIX ${ }^{e}$ siècle. Bien que la quantité de structures soit relativement limitée, l'interprétation de la carte archéologique montrerait à l'échelle de ce territoire une dynamique d'anthropisation assez «tardive », avec seulement trois sites associés au Néolithique (un dépôt de mobilier, un mégalithe ainsi qu'un habitat) avant de s'intensifier durant la période protohistorique par la présence de trois sites de hauteur présentant des fortifications talutées, d'une superficie souvent égale ou inférieure à 2 ha. Quelques monnaies romaines sont découvertes sur le territoire et une voie dite «romaine » traverse la forêt du nord au sud sur près de $8 \mathrm{~km}$. La présence humaine s'intensifie à la fin du haut Moyen Âge comme en attestent sept églises, associées depuis peu au style roman précoce (Wasylyszyn, 2007 ; Blanchard, 2018), situées dans des communes limitrophes, sans compter trois mottes castrales qui ceinturent la forêt (Sées, La Lande-de-Goult et La Roche-Mabile). De même, six ermitages, prieurés et chapelles s'inscrivent sur ce territoire forestier. Les témoignages d'activités humaines se poursuivent à l'époque moderne avec l'installation de sites proto-industriels comme les forges $(n b r e=3)$ et verreries $(n b r e=5)$, situées en lisière ou dans des enclaves forestières. Cette très faible densité de structures archéologiques n'est pas un cas à part et se rencontre systématiquement dans l'étude des espaces forestiers français qui, dans 
la plupart des cas, restent très peu documentés. D'après Murielle Georges-Leroy et al. (2009), l'absence de vestiges en contexte forestier traduit davantage une absence de prospection qu'une absence réelle, d'autant plus que l'intérêt de l'archéologie pour les forêts reste relativement récent (Desbordes, 1973).

\section{Déficit d'informations pour le département de l'Orne}

9 De cette base de données assez mince, doit être tiré un premier constat que les rares chercheurs à s'être intéressés au territoire n'ont pas manqué de souligner (Houzard, 1986 ; 1980 ; Louise, 1988), à savoir que le département de l'Orne souffre d'un déficit d'information. Néanmoins, les rares études régionales optant pour une démarche transdisciplinaire se sont intéressées au rôle de la forêt dans l'espace et dans le temps. Une étude transdisciplinaire pionnière est due au géographe Gérard Houzard qui, dans les années 1980, développe une biogéographie historique en utilisant les archives historiques sur les forêts bas-normandes, incluant celles d'Écouves et d'Andaines. Portant sur l'influence des activités métallurgiques sur les massifs forestiers, cette étude propose une histoire moderne de la forêt, de 1666 au xix siècle. Son usage des sources reste toutefois limité au regard de l'importance du corpus disponible. S'il se restreint à quelques archives d'intérêt capital (recueil de cartes de 1780, procèsverbaux et projets d'aménagement conservés aux ADO, Houzard délaisse des sources importantes comme les cahiers de doléances de 1789, qui renferment de nombreuses descriptions de finages, des rapports des sociétés à la forêt ou encore le procès-verbal de 1667 qu'il ne cite qu'indirectement, par le biais d'autres auteurs. De même, l'historien Gérard Louise (1988) propose dans sa thèse sur la seigneurie de Bellême (qui s'étend sur l'actuel département de l'Orne), du xe au XIII ${ }^{\mathrm{e}}$ siècle, certains points de réflexion sur l'aspect forestier du paysage durant le haut Moyen Âge et le Moyen Âge classique. L'auteur reprend l'hypothèse de la forêt « primitive » (primaire) comme c'est souvent le cas pour les grands massifs domaniaux (Musset, 1961), faisant des forêts du Perche, d'Andaines et d'Écouves les reliques d'une "forêt frontière ». Mais sans analyses paléoécologiques pour étayer ses propos, Louise multiplie les hypothèses sans pouvoir les vérifier : « Malheureusement pour notre région, les sondages et les analyses polliniques publiées font défaut. » Nécessaire, l'analyse paléoécologique constitue l'une des approches envisagées pour déterminer la dynamique d'évolution de la végétation. Or, de la même façon, les données paléo-environnementales sont rares dans cette partie de la Normandie par comparaison à ce qui existe en Bretagne ou dans le centre du Bassin parisien.

\section{Les données paléoécologiques disponibles}

Découvert fortuitement lors d'un chantier à proximité de la motte castrale de La RocheMabile (sud-ouest du massif forestier), un atelier de poterie (xII ${ }^{\mathrm{e}}$ siècle) a donné lieu à une analyse anthracologique (Marguerie, 1991). D'après celle-ci, il est permis de dire qu'existait dans les environs "une zone forestière assez dense " au Moyen Âge classique. Il faut attendre 1999 pour obtenir dans le nord de la Mayenne une étude globale sur l'évolution de la végétation sur la très longue durée (Barbier, 1999) réalisée à partir d'un corpus de tourbières dont deux sont situées à une quinzaine de kilomètres à l'ouest de la forêt d'Écouves. D'après Delphine Barbier, les premières traces d'anthropisation apparaissent dans la région à la fin du Néolithique final ou au début 
de l'âge du Bronze, comme en témoigne l'apparition de céréales accompagnées d'un taux assez élevé d'essences adventices comme l'oseille (Rumex) et le plantin (Plantago). À cette période la couverture forestière alterne successivement avec des peuplements composés majoritairement de chêne (Quercus) et de hêtre (Fagus S.) avec la présence de charme (Carpinus), de noisetier (Corylus) et de tilleul (Tilia). Des défrichements apparaissent à l'époque gallo-romaine dans l'un des deux sondages. Les forçages anthropiques (perturbations écosystémiques) semblent s'accélérer dans le temps avant de s'amenuir à partir du Bas-Empire. Le couvert forestier se dégrade à nouveau au début des Temps modernes avec un pic au cours des XVII ${ }^{e}$ et XVIII ${ }^{e}$ siècles. Si ces données permettent de documenter l'évolution du paysage depuis les premières occupations humaines, les sondages présentent deux hiatus sédimentaires, d'environ 3800 ans pour le premier et d'environ 300 ans pour le second qui s'expliquent par l'exploitation des tourbières à plusieurs reprises. Afin de préciser ces données sur le territoire étudié et de localiser l'implantation de nouveaux sondages et de contextualiser ces futures analyses paléoécologiques en forêt d'Écouves, un recours aux sources anciennes est donc nécessaire.

\section{Matériels et méthodes : les sources mobilisées}

\section{Les sources historiques}

\section{Cartes et documents anciens}

11 La majorité des archives consultées (figure 2) est conservée aux ADO, aux Archives départementales de Seine-Maritime (ADSM) ainsi qu'à la BNF. Il s'agit pour l'essentiel de documents administratifs émanant des Eaux et Forêts du XVII ${ }^{\mathrm{e}}$ siècle au XIX ${ }^{\mathrm{e}}$ siècle, à commencer par des procès-verbaux (P.-V.), et notamment le P.-V. de bornage, de visite et d'arpentage de 1667 (ADO, cote: 62B1). Effectué en prévision de l'ordonnance de Colbert qui réforme la gestion des bois du domaine, le $\mathrm{P}$.-V. de 1667 conserve de très riches informations concernant la forêt d'Écouves, comme les peuplements forestiers et leur âge en cette fin du xviI ${ }^{e}$ siècle, la toponymie en usage, la couleur des horizons superficiels, les aliénations de pièces de terre ainsi que les noms et professions des propriétaires ou exploitants limitrophes. 
Figure 2. Répartition des sources (manuscrites et planimétriques) disponibles sur la forêt d'Écouves dans les différents services d'archivage

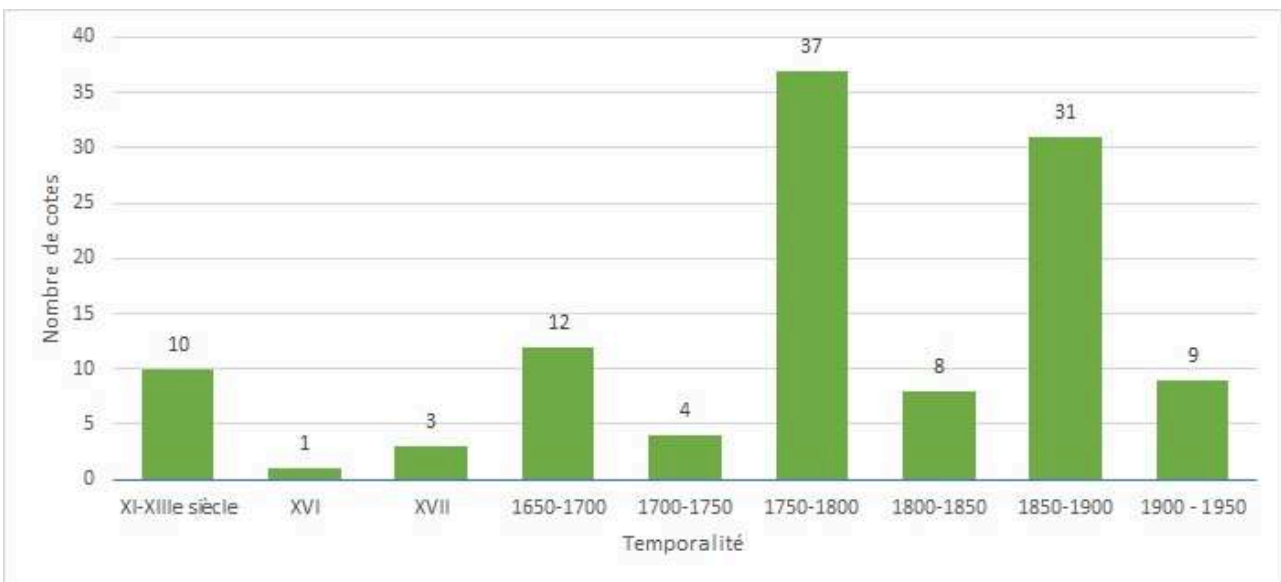

Source : Nicolas Blanchard.

Cette dernière information est importante puisque les propriétaires présentent à l'administration en cas de litige ou de soupçon d'usurpation, l'acte de propriété de leur parcelle, situant chronologiquement l'ouverture de certaines parcelles comme il suit :

«Est aussy comparons le sieur du Coudray lequel nous a dit que luy et ses predecesseur ont jouy de tout temps immemorial desdits heritages ce qui parrois

[...] avoient esté fieffé des le temps des ducs d'Alençon ", soit au début du $\mathrm{Xv}^{\mathrm{e}}$ siècle.

Le P.-V. de bornage, de visite et d'arpentage de 1667 constitue la base principale du corpus d'archives utilisé. Il s'associe avec un plan en deux parties (figure 3), daté de 1674 (BNF : cote GE CC-4945 [53RES] et GE CC-4945 [50RES]) qui constitue la représentation géographique la plus ancienne de la forêt d'Écouves connue à ce jour. On y remarque, sur les lisières dessinées, des espaces délimités par des pointillés rouges correspondant, d'après les informations mentionnées sur le document, à des « pièces de terre » réunies à cette période à l'espace forestier.

Figure 3. Plan de la forêt royalle d'Escouves par Jean Fleury, détails
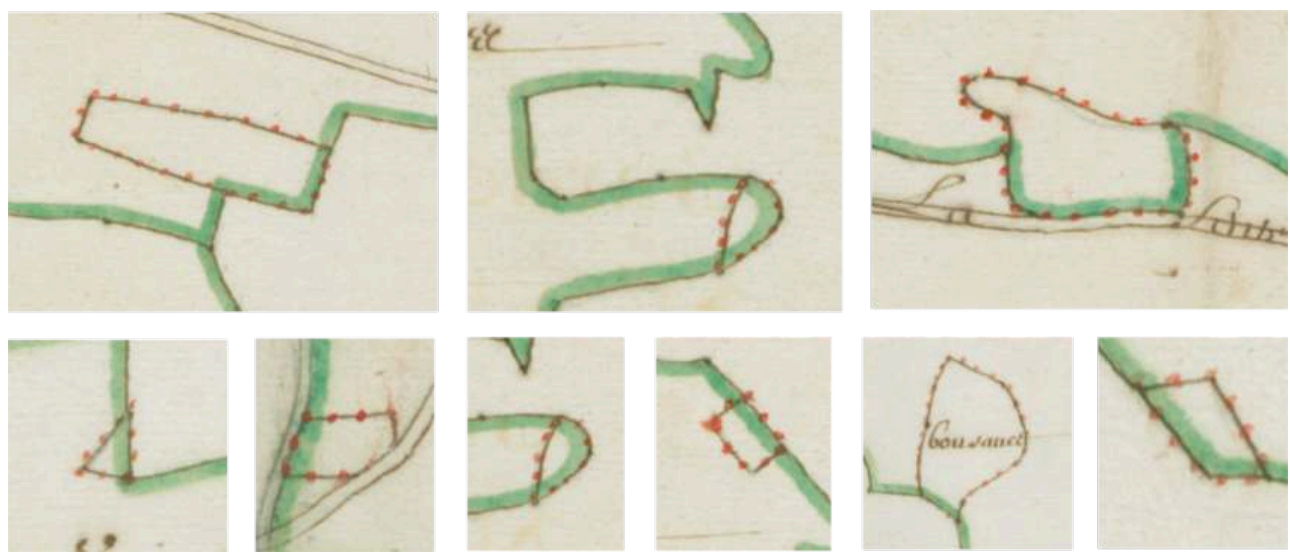

Source : Source gallica.bnf.fr/BNF GE CC-4945 (53RES) et GE CC-4945 (50RES).

Les autres documents consultés regroupent le projet d'aménagement de la forêt d'Écouves de 1781 (ADO, 62B38) ainsi que deux recueils de plans à l'échelle 1/14 000 de la même époque (ADO, 62B40 ; 62B41). À ces sources, s'ajoutent le cadastre du xIX siècle 
(1/4 000) des communes concernées et, lorsqu'ils sont disponibles, les plans par masses de cultures. Les plans (sans échelle) de plusieurs bois limitrophes comme le bois de Goult (ADO, cote : 66B17), de Rochelie (ADO, cote : 66B21; 66B22) et de Chaumont (ADO, cote : 66B19; 66B20) datant de la période révolutionnaire ont également été mis au jour et utilisés dans le cadre de l'étude. En outre, les microtoponymes en usage et ceux apparaissant sur les cartes anciennes et pouvant être interprétés comme des interactions entre les sociétés et leur environnement ont également été relevés. Ainsi, tous les termes associés à des ouvertures du milieu forestier comme Arcis, les Terres rouges, les Terres noires, les Essarts, la Prise ont été de cette façon répertoriés (WirthJaillard, 2019). De même, les anthrotoponymes présentant des suffixes en -ière ou -erie (la Picardière, les Ragottières ou les Potteries), également associés à des entreprises de défrichement du Moyen Âge classique (Duparc, 1964 ; Louise, 1988 ; Watteaux, 2012), ont été associés à cette démarche. Le linguiste Lepelley (1999) indique que ces toponymes sont construits d'un préfixe composé d'un patronyme et d'un suffixe en ière ou en -erie. Dans les deux cas, le toponyme vient inscrire dans le territoire une entreprise agricole familiale (d'où la présence du nom de famille dans le nom du lieu qu'il désigne), dont l'installation précède un défrichement effectué au cours du Moyen Âge classique, car il ne s'appuie plus sur une base latine. Les toponymes en être- (l'ÊtreRagaine), quant à eux, sont associés à des installations beaucoup plus tardives, autour des XIV et XVe siècles (Lepelley, 1999).

\section{Digitalisation des cartes anciennes et SIG}

15 Si la carte en deux parties de $1667(1 / 22000)$ présentait de nombreuses imprécisions pour pouvoir être géoréférencée et projetée sur la carte actuelle de la forêt d'Écouves, les cartes conservées aux $\mathrm{ADO}$ présentaient toutes les qualités nécessaires à leur digitalisation (numérisation en HD, contours précis, une échelle au 1/14 000, repères spatiaux facilement identifiables). Le document 62B41 a été privilégié pour la qualité de l'information qu'il renferme: localisation et numérotation des bornes de lisières, figuration des principaux chemins et voies de communication, etc. Construits la plupart du temps en deux parties, les plans des gardes (unités de gestion forestière) sont préalablement détourés, rassemblés et réorientés sous le logiciel de dessin vectoriel Inkscape (Rochel, 2016). Une fois l'opération effectuée, l'image est intégrée sur le logiciel QGIS via le plug-in géoréférencement. Un maximum de 10 points de contrôle sont alors calés depuis le raster sur le parcellaire forestier actuel avec des repères fiables, comme les lisières, les carrefours et les routes qui formaient à l'époque les limites des unités de gestion forestière (gardes, triages et ventes). Une transformation polynomiale de degré 2 a été privilégiée pour la qualité des résultats obtenus, couplée à une méthode de rééchantillonnage linéaire. De la même façon, les plans (1770) de bois privés limitrophes de la forêt domaniale ont été géoréférencés et digitalisés afin de préciser l'ancienneté du massif dans son ensemble et de mesurer son étendue aux périodes les plus reculées. Toutes les informations disponibles sur les cartes et permettant de renseigner des activités ou des structures potentielles (fontaines, occupations, toponymes, voies) ont été vectorisées et intégrées sous SIG par couches (points, lignes et polygones). Les toponymes, intéressant l'étude et parfois précisés par le cadastre du XIX ${ }^{e}$ siècle (1808-1818) conservé aux ADO, ont par ailleurs été intégrés sous SIG dans une couche point après l'intégration de la carte topographique de IGN $(1 / 25000)$. Ce recensement toponymique a donné lieu à une représentation spatiale des 
lieux-dits en distinguant trois classes (-erie, -ière et être-) avant de les relier arbitrairement sous forme d'isolines ou iso-toponymes. Des allers-retours entre les différents supports, images satellites/images aériennes à différentes époques, ont donné lieu à une lecture précise de l'ancienneté des masses boisées.

\section{Lecture et croisement des sources}

\section{Les parcellaires agricoles anciens}

Afin de révéler des parcellaires anciennement agricoles aujourd'hui sous couvert forestier, le P.-V. de bornage de 1667, la carte en deux parties de 1674, ainsi que les plans de la forêt d'Écouves de 1781, ont été croisés (figure 4). Partant des zones cerclées par des pointillés rouges (figure 3) et présentées comme " pièces de terre aliénées » sur les plans de 1674, il a été opéré un report de leur localisation donnée sur le plan de 1674 sur celui de 1781, en utilisant les numéros de bornes recensées. Obtenant le numéro de la borne la plus proche de la structure, il ne restait plus qu'à retrouver dans le corps du P.-V. le paragraphe correspondant à la parcelle aliénée en question, fournissant ainsi les informations souhaitées. Deux des anciens parcellaires ainsi identifiés, bien que réunis à la forêt d'Écouves en 1667, correspondent encore aujourd'hui à de petites parcelles cadastrales. De fait, si de nombreuses unités foncières intraforestières correspondent le plus souvent à des peuplements de résineux mis en culture récemment $^{3}$, certaines parcelles cadastrales, situées en lisière de forêt, non mentionnées dans les archives, ont également été associées à la phase de prospection archéologique afin de les assimiler (ou non) au corpus des parcellaires anciens conservés sous couvert forestier.

Figure 4. Processus de lecture des sources historiques
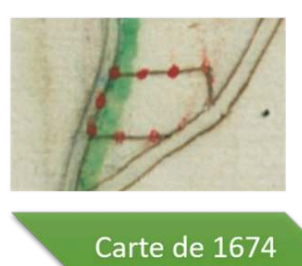

(localisation des parcelles)

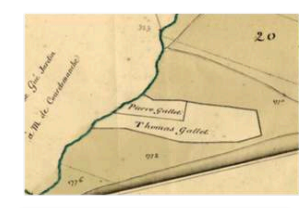

Plans de 1780

( $n^{\circ}$ des bornes)

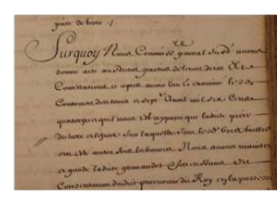

P.-V de 1667

(informations sur les parcellaires)

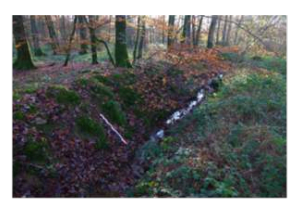

Vérifications de terrains

Exemple avec la parcelle des « Landettes » située à Saint-Nicolas-des-Bois.

Sources (de gauche à droite) : gallica.bnf.fr/BNF GE CC-4945 (53RES) ; ADO (62B40) ; ADO (62B1) ; Nicolas Blanchard.

\section{Les zones à mollières}

Le projet d'aménagement de 1781 (ADO, 62B38) mentionne à de nombreuses reprises le terme de « mollière » très probablement associé à des lieux humides :

«Au pied de ces montagnes, et même à mi-côte, on trouve des places très aquatiques qu'on appelle mollières, il en sort des ruisseaux considérables en hiver, qui sont souvent secs en été. »

Le terme dérive du latin molis, -ere, -fico, -filius et désigne un corps: mou, tendre, souple et moelleux (Gaffiot). Pour chaque mollière mentionnée dans le P.-V, une entité 
figurée en bleu était représentée dans le triage associé dans le recueil de plan de 1781 (ADO, cote 62B41). Le géoréférencement de l'ensemble des cartes a permis, grâce à la reprojection sur la carte actuelle, de localiser et de confirmer in situ le caractère tourbeux des «mollières ». L'intérêt de ces milieux humides pour l'étude de l'évolution de la végétation réside en ce que les tourbières conservent de nombreux macrorestes organiques incluant pollens, graines, charbons de bois, ou encore restes ligneux. En tout 33 mollières ont été identifiées de cette façon, dans le but d'entreprendre des analyses paléoécologiques (palynologie, anthracologie, datations).

\section{Prospection pédestre en milieu forestier}

\section{Principes et méthodes}

Les principes de la prospection archéologique en milieu forestier ont été établis pour la première fois par Jean-Michel Desbordes en 1973. Elle se distingue en de nombreux points de la prospection en milieu ouvert dans la mesure où elle s'effectue dans des espaces pour la plupart mal documentés, sans renfort d'images aériennes et d'indices phytographiques mais également par la difficile perception des structures archéologiques qu'implique une végétation permanente. Seule l'imagerie Lidar est en mesure de mettre en évidence de façon systématique des anomalies ponctuelles, surfaciques ou linéaires en milieu forestier. L'absence d'une vision panoramique oblige le prospecteur à parcourir de vastes étendues sans certitudes préalables. Dans la mesure où en 1667 l'administration des Eaux et Forêts demande expressément le comblement des fossés (ADO, cote 62B1), le but de la prospection avait pour objet de confirmer ou non la présence de structures limitantes apparentes, matérialisant les parcellaires identifiés dans les archives. Les coordonnées GPS des structures identifiées ont été relevées au GPS topographique (Garmin Oregon $450 \mathrm{~T}$ ) et intégrées par la suite sous SIG. La prospection s'est également étendue aux zones tourbeuses identifiées sur les cartes anciennes (figure 5), avec dans un premier temps des sondages à la tarière avec description des profils pédologiques, de façon à : 1) mettre en évidence des séquences de sédimentation organique importante; 2) de dater par radiocarbone les éléments organiques (charbons, graines, macrorestes ligneux), plus particulièrement localisés à la base des sondages. 


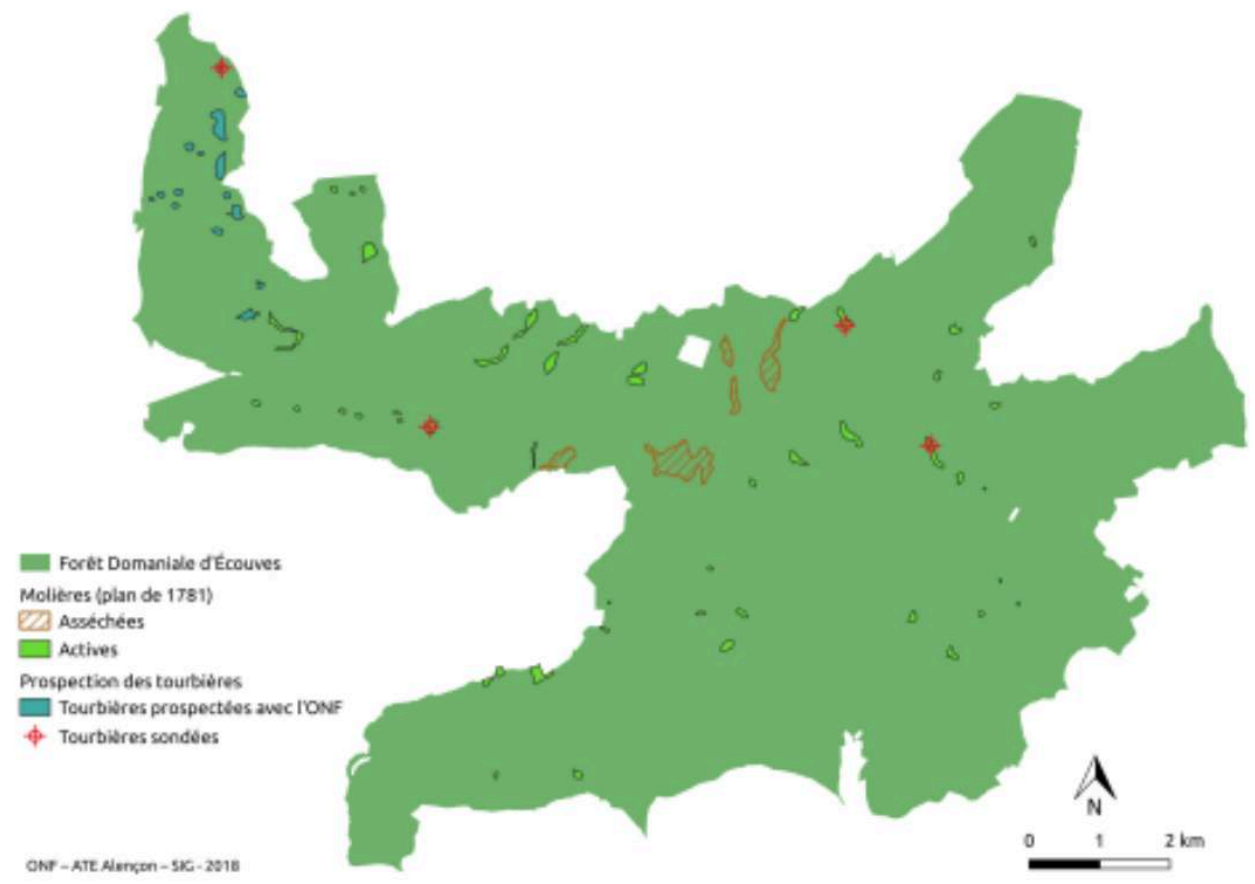

Source : Nicolas Blanchard/ONF.

\section{Relevés botaniques et formations végétales anthropogènes}

Une autre singularité de l'archéologie en sous-bois, révélée à de nombreuses reprises (Desbordes, 1973 ; Dupouey, 2007 ; Doyen et al., 2004), se rencontre dans la végétation observée au niveau des contextes archéologiques. Déjà dans son principe d'archéologie forestière, Desbordes (1973) précise que les anomalies d'origine anthropique en contexte forestier "sont presque toujours associées à des anomalies botaniques". Certaines essences comme le fragon (Ruscus aculeatus), sur lesquelles les publications abondent, sont aujourd'hui reconnues à la fois comme essence anthropogène (Couderc, 1985) et archéo-indicateur (Lemouland, 2009). De fait, pour documenter au mieux ces structures, il a été décidé de recenser les principales essences associées aux structures rencontrées sur le tapis végétal de l'ensemble des parcelles, afin d'apporter une large contribution à l'analyse fonctionnelle de ces sites. Dans la mesure où ces derniers sont pressentis comme étant d'anciens parcellaires à vocation agricole, un impact significatif sur les propriétés physico-chimiques des sols, identifiables par la présence ou l'absence de certains végétaux, est attendu. Qu'il s'agisse de proximité avec des contextes archéologiques (rejets/habitats) ou de parcelles anciennement cultivées, les conditions phyto-écologiques comme les $\mathrm{pH}$ évoluent à la faveur de cortèges spécifiques. De fait, la prospection a également donné lieu à l'analyse complémentaire des $\mathrm{pH}$ eau à l'intérieur et à l'extérieur de chaque structure identifiée à l'aide d'un $\mathrm{pH}$ mètre électronique portable de type $\mathrm{Exstik}^{\mathrm{TM}}$. Au total, 52 analyses $\mathrm{pH}$ ont été réalisées, soit 4 par parcellaire. 


\section{Résultats : l'anthropisation préforestière et les dynamiques d'évolution des paysages forestiers}

\section{Caractérisation des formations parcellaires}

21 Sur place, on observe que toutes ces anciennes parcelles sont boisées et leur délimitation fossoyée correspond à celle de la forêt avant 1667 (figure 6). D'emblée, on remarque de nombreuses ressemblances dans la morphologie des parcellaires anciens. Premièrement, toutes ces structures sont signalées dans les archives comme étant "enclavées dans la forêt ». Elles présentent un aspect longiforme et pénétrant en direction du cœur forestier. Dans la majorité des cas, les parcelles ont une forme légèrement conique dont le côté le plus large est orienté vers le cœur du massif.

Figure 6. Limite forestière en contexte forestier signalant un ancien parcellaire agricole

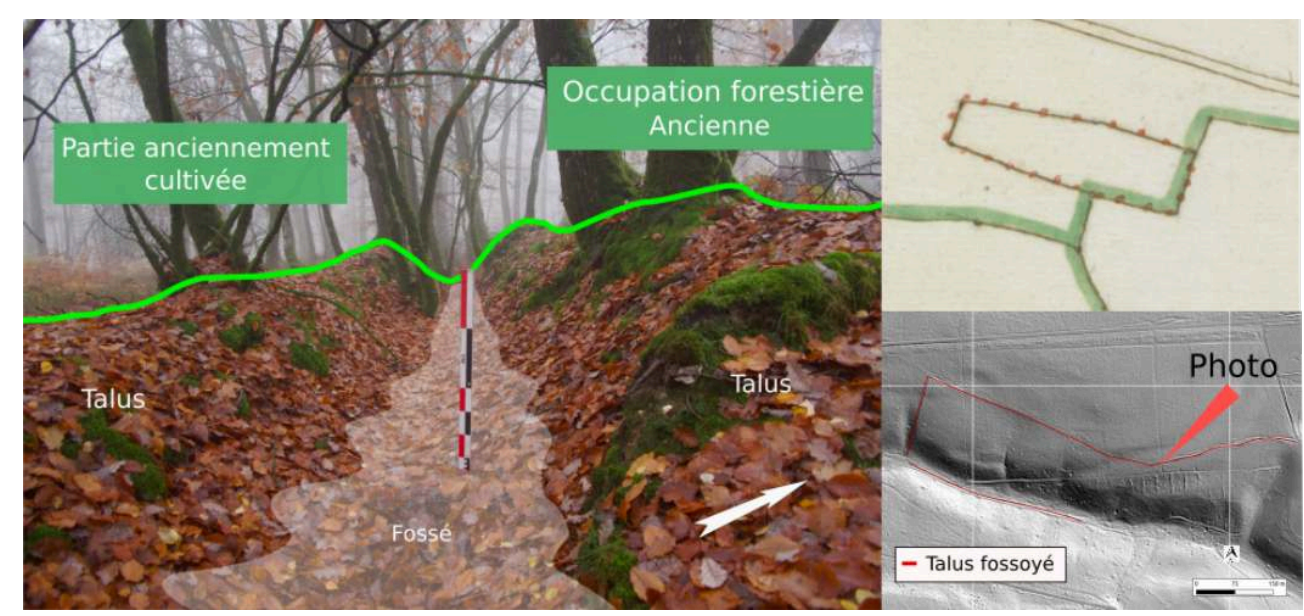

À gauche : talus fossoyé délimitant un ancien parcellaire forestier de la forêt royale ; à gauche du talus, la parcelle du val Roger mentionnée en 1667 comme étant « partie en labour et place vaine et vagues en partie en bois ». À droite : sol forestier en place depuis 1667 ; en haut à droite : Plan de la forêt royalle d'Escouves (détail) par Jean Fleury.

Sources : Nicolas Blanchard à gauche; gallica.bnf.fr/BNF GE CC-4945 (53RES) en haut à droite ; image Lidar (Drac/ONF) en bas à droite.

Ce même côté s'appuie systématiquement sur une route ou un ruisseau dont il est perpendiculaire, ce qui d'après Soyer (1970) représente un signe caractéristique d'un défrichement, dans la mesure où le chemin précède nécessairement l'ouverture du milieu. De fait, l'orientation des parcelles semble principalement découler de l'architecture $\mathrm{du}$ réseau viaire ou hydraulique préexistant. L'interprétation de l'imagerie Lidar permet également de préciser les formes et contours de ces parcellaires mais également d'en découvrir 17 autres, présentant une morphologie similaire et pour lesquels aucune documentation n'a été mise au jour. 


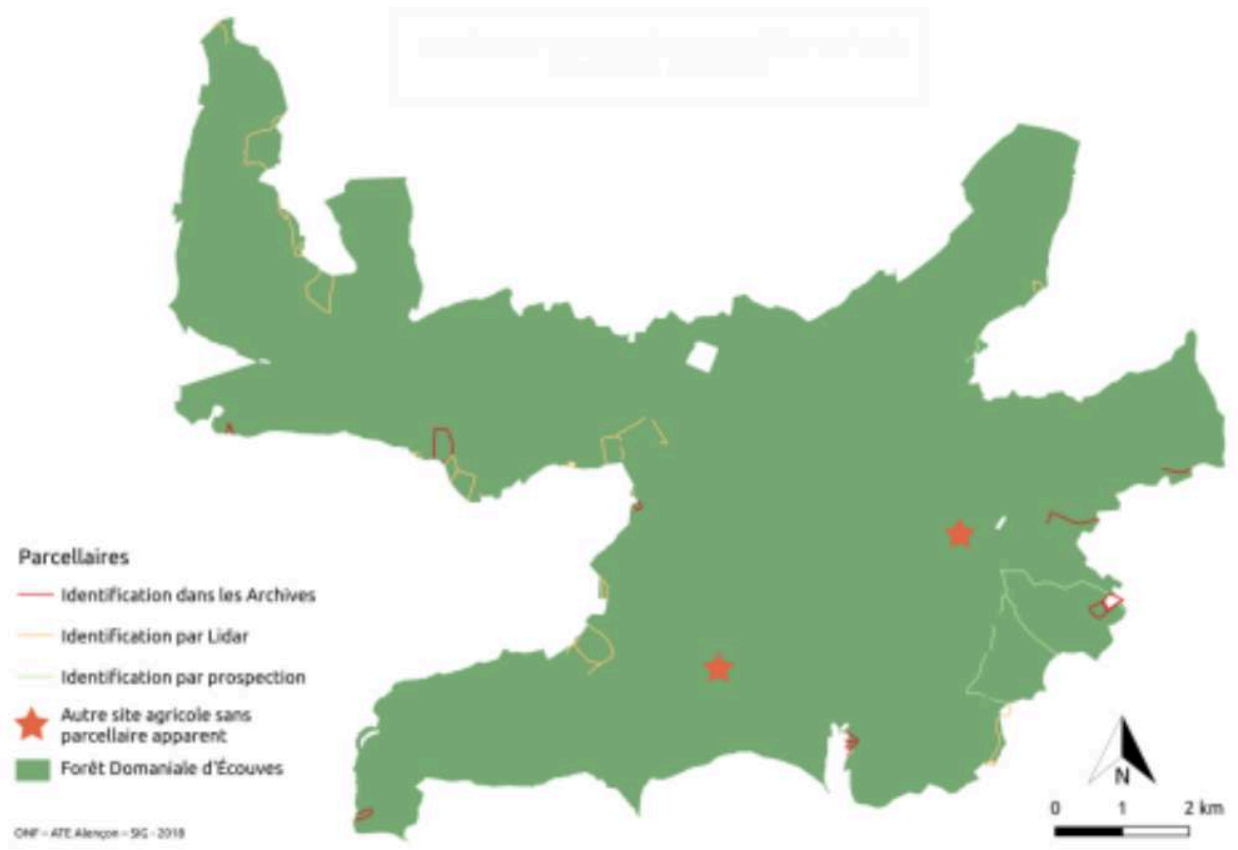

Source : Nicolas Blanchard/ONF.

D'après les archives consultées et notamment le P.-V. de 1667, la majorité de ces ouvertures a été pratiquée avant les $\mathrm{Xv}^{\mathrm{e}}$ et $\mathrm{XVI}^{\mathrm{e}}$ siècles. Ces parcellaires (figure 7) rejoignent le corpus de deux enclaves forestières qui correspondent à deux anciens ermitages, connus localement sous les noms de champs de Poitou et de l'ermitage de Vingt-Hanaps. D'après un P.-V. de $1665^{4}$, sont rattachés à ce premier site «tant en taillis qu'en terres labourables environ trente arpents ». Le second est identifié dans les sources comme "terram de Martellum-Vedosium» au cours du $\mathrm{XI}^{\mathrm{e}}$ siècle. Ces enclaves sont d'ailleurs visitées par Bernard-Hector de Marles dans le P.-V. de visite de 1667. L'auteur y note la présence de haies relictuelles et des traces de labours :

« Nous avons passé le triage de dessous l'ermitage par une place vague que l'on a dit appartenir aux religieux de Saint-Martin de Sées, en nature d'herbage qui parrait néanmoins par les sillons avoir été autrefois en labour, sur laquelle il y a deux bâtiments, l'un servant de chapelle, l'autre de maison manable. »

En comptant les deux ermitages, 13 parcellaires agraires ont été recensés dans les documents d'archives. Néanmoins, si les archives attestent d'une activité agropastorale, aucune trace de labour n'a été observée à l'intérieur de ces parcelles à ce jour.

\section{Des parcellaires identiques en contexte extra-forestier}

La définition d'une morphologie typique des parcelles anciennement agricoles réunies au cœur de la forêt au XVII siècle nous a conduits à vérifier l'ensemble des parcelles agricole situées en lisière du massif forestier en confrontant images satellites, photographies aériennes des années 1950 et cartes anciennes. Il en ressort que de nombreuses parcelles se rapprochent de cette «morphologie type » et s'apparentent à des formes parcellaires de défrichement résilientes. Leur morphologie s'explique soit 
par leur proximité directe encore effective avec une lisière forestière sans avoir été aliénée à l'espace forestier en 1667, soit par leur ancienne proximité avec une lisière disparue après défrichements tardifs. C'est le cas notamment au nord-ouest du massif où peut s'observer ce type de parcellaire. De même, au nord et au nord-est du massif (Saint-Chistophe-le-Jajolet), fonctionnant sur la base d'un réseau viaire en appui dont la parcelle de défrichement est perpendiculaire, on observe des zones de défrichement intenses qui cette fois-ci semblent planifiées, organisées non plus à l'échelle d'une parcelle mais d'un terroir complet (friche forestière, bruyère...). Le cas des bois de Montgommeries (nord-ouest) (figure 8) est assez intéressant puisqu'une grande partie a été défrichée au cours du XIX ${ }^{e}$ siècle. Cependant, les anciennes lisières fonctionnent encore actuellement comme limites communales et s'observent dans la trame parcellaire puisque les parcelles qui jouxtaient anciennement la lisière n'ont pas été remembrées et ont conservé leur morphologie d'origine (primoforme).

Figure 8. Évolution parcellaire du lieu-dit l'Être-Logeard, situé sur l'ancienne lisière du Bois des Montgommeries

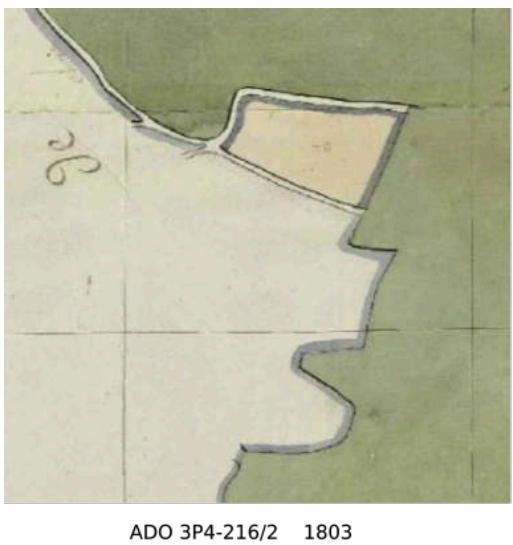

Source : ADO

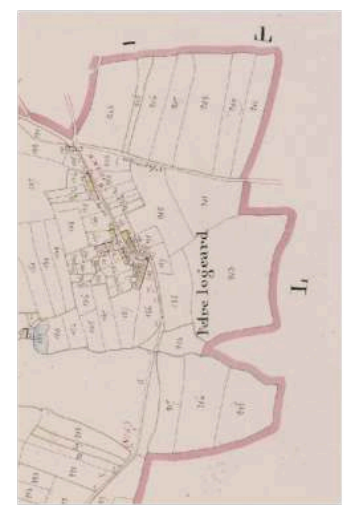

ADO 3 P2-216/10 1818

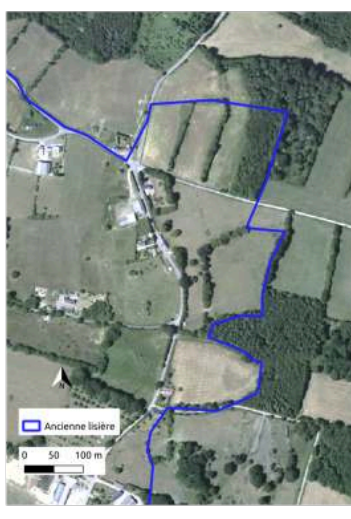

Image satellite

\section{Signature botanique et indices d'anthropisation des milieux}

Si la strate herbacée d'Écouves se caractérise majoritairement par la présence d'une végétation acidicline et acidiphile, les parcelles anciennement cultivées (anthroposols) présentent systématiquement un cortège floristique beaucoup plus riche en espèces (Doyen et al., 2004; Lemouland, 2009). De même, l'interprétation des données autécologiques du cortège rencontré indique un sol plus ou moins riche en éléments nutritifs, en bases comme en azote pour certaines d'entre elles, incluant le sureau (Sambucus nigra), le lamier jaune (Lamium galeobdolon), ou encore l'arum tacheté (Arum maculatum). En outre, les essences observées (figure 9) signalent un pH basique à neutre, voire neutre à faiblement acide, comme le montre la présence d'euphorbe des bois (Euphorbia amygdaloides), de stellaire holostée (Stellaria holostea), de mélique uniflore (Melica uniflora), de rosier des chiens (Rosa canina), d'aspérule odorante (Galium odoratum), ou encore de ronce (Rubus fruticosus), ce qui diffère totalement $\mathrm{du} \mathrm{pH}$ habituellement rencontré en forêt d'Écouves, majoritairement acide à très acide (Jabiol, 1985). 
Figure 9. Répartition en pourcentage des essences rencontrées dans 12 parcellaires anciens (XIVE$\mathrm{xVl} l^{\mathrm{e}}$ siècle)

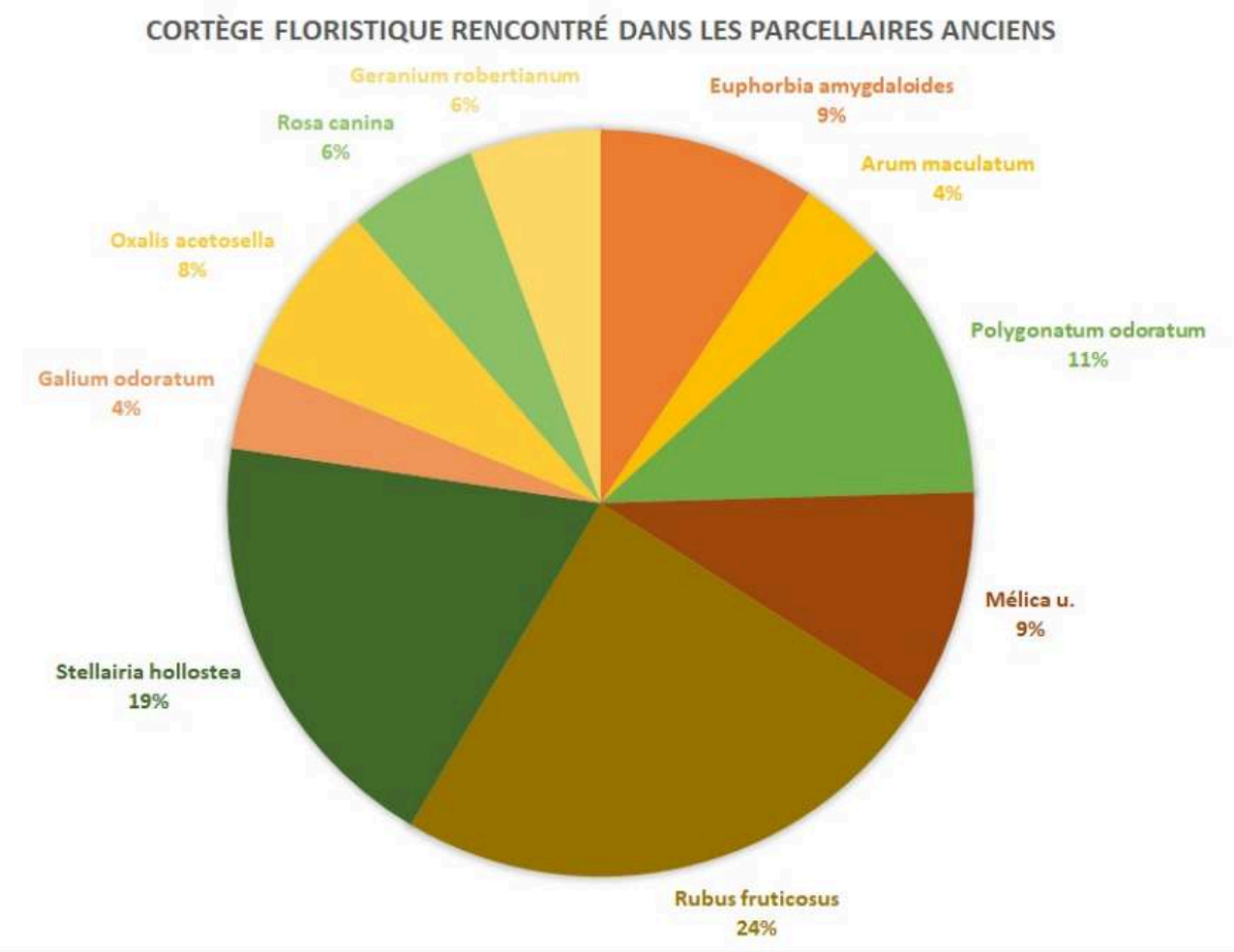

La répartition s'exprime par taux de présence sur l'ensemble des parcellaires observés.

Source : Nicolas Blanchard.

Cette diversité du cortège floristique s'apparente de fait à un facteur anthropique dans la mesure où ces parcelles ont probablement été amendées, soit volontairement par des pratiques agricoles, soit indirectement par la présence d'animaux domestiques (présence d'azote). L'ensemble des analyses $\mathrm{pH}$ effectuées au sein des parcellaires agraires identifiés (52) donne un $\mathrm{pH}$ moyen de 6, avec un minimum de 3,5 et un maximum relevé de 8 . Dans notre cas, seules 8 analyses sur 52 (soit $15 \%$ ) présentent un $\mathrm{pH}<5$, ce qui diffère largement des observations faites par Jabiol (1985). Seul l'ensemble des $\mathrm{pH}$ relevés à l'extérieur des parcellaires se sont révélés $<5$. De fait, les pratiques agricoles anciennes (apport d'éléments organiques) ont profondément amélioré la nature du sol, modifiant à la fois le pH (Lemouland, 2009) comme la composition de la strate herbacée. Certains parcellaires encore exploités jusqu'au début $\mathrm{du}$ siècle dernier présentent des traces de haies (alignements de souches), d'épierrements sur lesquels se rencontre encore du barbelé, jalonné d'arbres têtards. La réalisation de l'inspection des lisières actuelles de la forêt domaniale et de ses anciennes parties aliénées au XIX ${ }^{e}$ siècle (Barre de Chahains, la Petite Gastine) a, de même, révélé un certain nombre d'arbres têtards et de haies plessées sur les bords extérieurs des lisières. 


\section{Discontinuités et progressions de la surface boisée}

\section{D'après la toponymie}

Si la prudence est de mise concernant l'usage des toponymes en archéologie ou toute approche géohistorique (Zadora-Rio, 2001), l'abondance des références concernant les anthrotoponymes en -ière et -erie (Duparc, 1964 ; Louise, 1988 ; Watteaux, 2012) nous a tout de même invités à considérer la question dans le cas du massif d'Écouves. En tout, 230 toponymes formés d'un suffixe en -ière ou -erie et un préfixe en -être ont été comptabilisés sur un ensemble de 22 communes limitrophes. En comparaison, un territoire non forestier de 22 communes comprises entre les villes de Falaise (Calvados), Vimoutiers et Argentan (Orne), ne totalise que 31 toponymes en -ière ou -erie. Les isolines formées représentent «des lignes de front " pénétrantes et successives d'avancées sur le massif, dessinant parfois la forme des lisières actuelles, comme dans l'enclave de la commune du Bouillon (nord-est). De cette façon, les toponymes en -ière dessinent une couronne autour de l'actuelle plaine d'Alençon tout en se rapprochant progressivement des lisières actuelles du massif.

Figure 10. Carte de répartition des formations toponymiques en -ière, -erie et être- autour du massif forestier d'Écouves, 2019

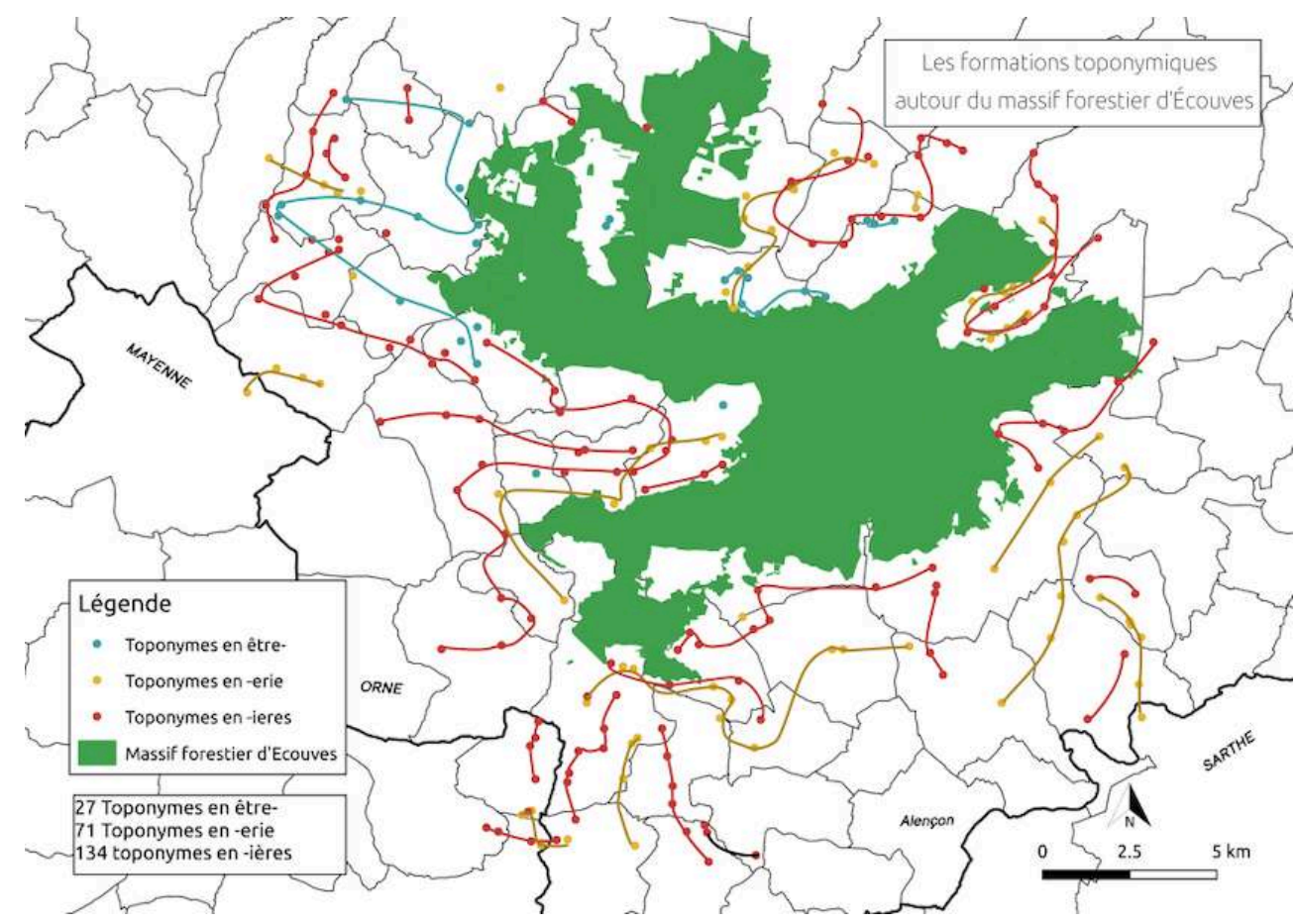

Sources : IGN/Nicolas Blanchard.

La datation relative des toponymes, avancée par Lepelley (1999), semble accréditée par la carte dans la mesure où les formations en -erie (traits jaunes) sont situées dans la plupart des cas à proximité directe de la lisière actuelle de la forêt d'Écouves (correspond aux défrichements les plus récents) alors que les anthrotoponymes en ière (traits rouges) s'inscrivent davantage dans les terres. Les toponymes en être(l'Être-Mathurin, l'Être-Savary) associés à des installations beaucoup plus tardives, 
autour des $\mathrm{XIV}^{\mathrm{e}}$ et $\mathrm{XV}^{\mathrm{e}}$ siècles (ibid.), expliquent une proximité plus prononcée aux lisières forestières (traits bleus).

\section{D'après l'analyse régressive}

L'usage des cartes anciennes avait permis à Houzard (2008) de réaliser des cartes des peuplements forestiers à différentes époques $(1780,1785,1923)$ même si ses travaux l'avaient amené à proposer l'hypothèse que la superficie du massif forestier d'Écouves avait peu bougé en cinq siècles. L'ensemble de ces documents nous a donné la possibilité de réaliser une carte retraçant les continuités et discontinuités de la couverture forestière sur la longue durée (figure 11). Les dernières investigations, ici présentées, étayent cette affirmation. La mobilisation et le croisement de l'ensemble des documents d'archives ont permis de mieux envisager l'évolution du massif sur les cinq derniers siècles. Les vérifications de terrain ont confirmé une intervention humaine presque omniprésente sur l'ensemble du massif (présence de parcellaires agraires, phytosociologie éloignée du cortège floristique classique, défrichements).

Figure 11. Ancienneté du sol forestier du massif d'Écouves et évolution de la surface boisée de la forêt domaniale de 1643 à 2004

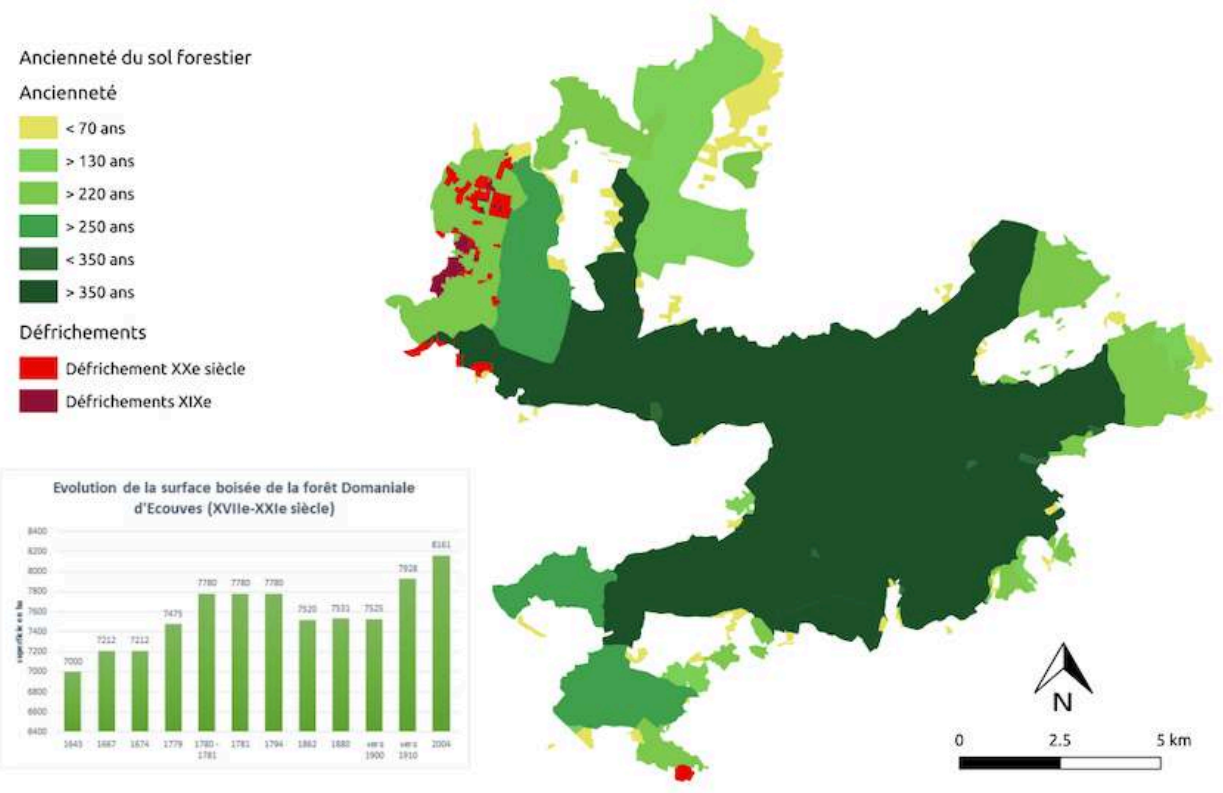

Sources : d'après les documents manuscrits et cartes anciennes, ONF, Nicolas Blanchard.

Si la majorité des unités forestières (bois privés, forêt domaniale) qui constituent le massif d'Écouves est mentionnée à des périodes bien antérieures ( $\mathrm{XII}^{\mathrm{e}}$-XIII ${ }^{\mathrm{e}}$ siècle) aux cartes anciennes (xvII ${ }^{\mathrm{e}}$-xIX $\mathrm{x}^{\mathrm{e}}$ siècle), la carte ci-dessus (figure 11) montre que $60 \%$ de la superficie actuelle du massif d'Écouves a conservé sa vocation forestière depuis plus de cinq siècles ( $\mathrm{XVII}^{\mathrm{e}}-\mathrm{XXI}{ }^{\mathrm{e}}$ siècle). Les seules incursions effectuées dans le cas de la forêt domaniale se concentrent sur les lisières avant de retourner à un état boisé après 1667. Passé le $\mathrm{xIX}{ }^{\mathrm{e}}$ siècle, seuls quelques défrichements forestiers effectués au cours de la période sont à mentionner dans les bois privés (nord-ouest). Excepté ces dernières soustractions forestières, la surface boisée de la forêt domaniale et de l'ensemble du massif n'a cessé d'augmenter depuis 1667. 


\section{Conclusion : l'espace forestier, une construction anthropique sur la longue durée}

bien souvent dans leur morphologie et leur peuplement les signes d'une anthropisation ancienne. Il reste cependant difficile de repérer ou d'identifier les structures ou indices d'anthropisation in situ, soit en raison d'une végétation trop dense, du fait de l'érosion naturelle (souvent limitée en contexte fermé et peu pentu), ou encore des remaniements anthropiques secondaires. C'est donc là tout l'intérêt du recours aux archives historiques grâce auxquelles il est possible de retrouver les anciens contours forestiers et de documenter leur évolution sur la longue durée (diachronie). En cela, la méthode qui consiste à croiser archives naturelles et sources historiques présente de nombreux avantages en permettant d'appréhender l'anthropisation de la forêt d'Écouves sur près de cinq siècles d'évolution.

Il n'en demeure pas moins que l'archive historique ne peut pas tout et que, passé quelques siècles, la lecture des documents se complexifie ou est rendue impossible par l'absence ou la disparition de sources pour certaines périodes. La singularité de l'information exploitable dans chaque document demeure propre au fonds d'archives investigué. Dans le but d'obtenir des informations biogéographiques et archéologiques, le croisement entre archives manuscrites et cartes anciennes est envisageable pour d'autres massifs forestiers, dans la mesure où la majorité des anciennes forêts royales a fait l'objet de nombreux procès-verbaux et de documents d'aménagement associés le plus souvent à des cartes anciennes. Dans le cas de la carte de la forêt d'Écouves de 1674 (figure 3), son auteur, l'arpenteur Jean Fleury, est également à l'origine de 93 plans de forêts royales (sa production s'étale de 1665 à 1687). Toutes sont numérisées et conservées à la BNF. Malheureusement, certains documents importants ont disparu, tels que les procès-verbaux des visites des forêts du dernier quart du XviI ${ }^{\mathrm{e}}$ siècle. C'est le cas par exemple pour les forêts domaniales de Senonches (Eure-et-Loir) et de Sillé-leGuillaume (Sarthe) pour lesquelles nous ne disposons plus de ces documents ${ }^{5}$. Pour la présente étude sur la forêt d'Écouves, les archives constituent le cœur de la méthodologie analytique, offrant la possibilité de documenter un certain nombre de structures archéologiques (les parcellaires agricoles anciens), de comprendre l'organisation de l'espace forestier à l'époque moderne et d'anticiper à la fois les données Lidar comme l'analyse paléoécologique.

Le croisement de l'ensemble des informations disponibles, issues des archives manuscrites, des cartes anciennes, des vérifications de terrain, est en mesure de mettre en place une carte d'ancienneté de l'état boisé très précise, notamment en s'appuyant sur des repères chronologiques antérieurs au minimum forestier, période généralement associée au milieu du XIx ${ }^{\mathrm{e}}$ siècle et qui correspond à la période durant laquelle la surface boisée était à son minimum.

La poursuite du travail va désormais consister à l'intégration des structures archéologiques découvertes par l'interprétation des données Lidar. En outre, l'analyse paléobotanique nécessite d'être approfondie et complétée par l'analyse palynologique. Enfin, l'objet de recherche que constituent les parcellaires agricoles nous invitera

Projets de paysage, 22 | 2020 
inévitablement à approfondir via une approche morphodynamique la question des défrichements qui reste encore assez mal documentée.

\section{BIBLIOGRAPHIE}

Barbier, D., « Histoire de la végétation du nord-mayennais de la fin du Weichsélien à l'aube du $\mathrm{XXI}^{\mathrm{e}}$ siècle. Mise en évidence d'un Tardiglaciaire armoricain. Interactions Homme-Milieux ", thèse de doctorat, sous la dir. de Lionel Visset, université de Nantes, 1999.

Blanchard, N., «Les espaces forestiers comme espaces culturels, l'exemple de la forêt d'Écouves », mémoire de master « Valorisation du patrimoine naturel et culture », université de RouenNormandie, 2018.

Couderc, J.-M., « Végétation anthropogène et prospection archéologique », Revue archéologique du Centre de la France, 1985, t. 24, fasc. 1, p. 53-61

Cubizolle, H., Sacca, C., Tourman, A., Porteret, J. et Thébaud, G., « Les tourbières du haut bassin versant de la Loire (Massif central oriental) », Norois, $\mathrm{n}^{\circ}$ 192, p. 95-115, 2004, mis en ligne en août 2008, URL : http://journals.openedition.org/norois/943 ; DOI : https://doi.org/10.4000/ norois.943

Dardignac, C., David, S., Le Jeune, Y., « Bercé avant la forêt, premiers résultats de l'exploitation des cartes anciennes et des données Lidar sur la forêt de Bercé (Sarthe) ", Revue forestière française, t. LXIX, 2017.

Desbordes, J.-M., « La recherche archéologique sous-bois », Revue archéologique de l'Oise, nº 3, 1973. p. 12.

Doyen, B., Decocq, G., Thuillier, P., « Archéologie des milieux boisés en Picardie », Revue archéologique de Picardie, $\mathrm{n}^{\circ}$ 1-2, 2004, p. 149-164.

Dumé, G., Gauberville, C., Mansion, D., Rameau, J.-C., Flore forestière française, t. I, Plaines et Collines, nouvelle édition, revue et augmentée, Paris, IDF- CNPF, 2018.

Duparc, P., "Les tenures en hébergement et en abergement », Bibliothèque de l'école des chartes, t. 122., 1964, p. 5-88, URL : https://doi.org/10.3406/bec.1964.449668

Dupouey, J.-L., Dambrine, E., Dardignac, C., Georges-Leroy, M., « La mémoire des forêts. Actes du colloque "Forêt, archéologie et environnement" 14 au 16 décembre 2004 », ONF, INA, Drac Lorraine, 2007, p. 294.

Georges-Leroy, M., Bock, J., Dambrine, E., Dupouey, J.-L., « Le massif forestier, objet pertinent pour la recherche archéologique. L'exemple du massif forestier de Haye (Meurthe-et-Moselle) », Revue géographique de l'Est, vol. 49, n 2-3, 2009, mis en ligne en avril 2013, URL : http:// journals.openedition.org/rge/1931

Houzard, G., « Dégradations et restauration en Écouves de 1666 à nos jours », dans Hommes et Terres du Nord. Actes du colloque «Du pollen au cadastre », 1986, n 2-3. p. 227-230, URL : https:// www.persee.fr/doc/htn_0018-439x_1986_num_2_1_2076 
Houzard G., «Les massifs forestiers de Basse-Normandie (Brix, Andaines et Écouves). Essai de biogéographie ", thèse de doctorat d'État, université de Caen, 2 vol., 1980, 667 p. ; publiée sous le titre Les Massifs forestiers d'Andaines et d'Écouves, Alençon, Société Historique et Archéologique de l'Orne (SHAO), 2008, 2 vol. , 208 p.

Jabiol, B., «Les stations forestières en forêt domaniale d'Écouves, (Orne) », Revue forestière française, t. XXXVII, 1985, p. 33-43.

Lemouland, Q., « Phytoarchéologie : impact d'occupations archéologiques sur la flore actuelle (historique, concepts, méthodes et études de cas) ", thèse de doctorat, sous la dir. de JeanLaurent Monnier, université de Rennes 1, 2009.

Lepelley, R., Noms de lieux de Normandie et des îles anglo-normandes. Introduction à la toponymie, Chamalières, Christine Bonneton, 1999.

Lespez, L., Germain, C., « Les paléoenvironnements de l'âge du fer en Basse Normandie : état des connaissances et problèmes posés » dans L'Âge du fer en Basse-Normandie, gestes funéraires en Gaule au Second âge du fer, actes du XXXIIIe colloque international de l'AFEAF, Caen, 20 au 24 mai 2009, Besançon, Presses universitaires de Franche-Comté, 2010.

Letacq, A.-L., Sainte-Catherine de Poitout à la Haie du Froust, Notice sur l'ancienne Chapelle de SainteCatherine de Poitou dans la forêt d'Écouves, Almanach des maisons Pierre Romet, 1931

Louise, G., « La seigneurie de Bellême (Xe-XIIe s.), étude historique et archéologique ", thèse de doctorat, sous la direction d'André Debord, université de Caen, 1988.

Marguerie, D., « Rapport d'étude anthracologique du site de la Grande Ouche à la Roche-Mabile », SRA, 1991.

Piriou J., « Tourbières et boisements dirigés : gestion, représentations et trajectoires géohistoriques. Application aux forêts d'Écouves (Basse-Normandie, France) et de Slieveanorra (Comté d'Antrim, Irlande du Nord) », thèse de doctorat, sous la direction de Jean-Paul Amat et Guy Lempérière, université Paris 4, 2013.

Piriou, J., Petit-Berghem, Y., Lempérière, G., Gramond, D., « Les mosaïques paysagères en forêt. Le cas des zones humides intraforestières du bois de Goult (forêt d'Écouves, Orne) ", Revue forestière française, t. LXII, 2011, p. 555-574.

ONF, « Document d'aménagement forestier de la forêt Domaniale d'Écouves, période d'application : 2004-2023 », ONF, 2004.

Musset, R., « Le nom et l'ancienne ceinture forestière du Pays de Caux ; les villages des défrichements médiévaux ", Norois, n 31, juillet-septembre 1961, p. 321-327, URL : https:// www.persee.fr/doc/noroi_0029-182x_1961_num_31_1_7199.

Rochel, X., « Paysages naturels, paysages construits, Géographie historique et usage des archives dans l'étude de paysages dits naturels (forêts, saltus) », HDR, université de Lorraine, 2016, 209 p.

Soyer, J., «La conservation de la forme circulaire dans le parcellaire Français, étude basée sur l'interprétation de photographie aériennes ", mémoire de photo-interprétation, SEVPEN, 1970.

Vigneau, T., « Biodiversité et archéologie : une étude interdisciplinaire en forêt de Rambouillet (Yvelines, France) », dans « La mémoire des Forêts », actes du colloque « Forêt, archéologie et environnement », ONF/Inra/Drac Lorraine, 2004, p. 163-171.

Wasylyszyn, N., « Inventaire et observation sur les églises romanes précoces de Haute-Normandie $\left(\mathrm{x}^{\mathrm{e}}-\mathrm{XI}^{\mathrm{e}}\right.$ siècle) », Haute-Normandie Archéologique, $\mathrm{n}^{\circ}$ 12, 2007, p. 75. 
Watteaux M., «Lecture archéologique d'un bocage vendéen ", Annales de Bretagne et des Pays de l'Ouest, 2012, p. 57-80 ; mis en ligne en juin 2014, URL http://journals.openedition.org/abpo/ 2395 ; DOI : https://doi.org/10.4000/abpo.2395

Wirth-Jaillard, A., « Nommer les défrichements », dans Bépoix, S. et Richard, H., La Forêt au Moyen Âge, Paris, Les Belles Lettres, 2019.

Zadora-Rio, E., « Archéologie et toponymie : le divorce ", Les Petits Cahiers d'Anatole, n 8, 2001.

\section{NOTES}

1. Nemore scoparum que l'on peut traduire par « la forêt buissonnante ».

2. Le livre de Marie d'Espagne datant de 1348 présentait l'inventaire des bois du domaine de la comtesse d'Alençon. Le livre a disparu dans un incendie.

3. Information donnée oralement par Lionel Huchette, chef de l'unité territoriale d'Alençon.

4. Mentionné dans Letacq A.-L., 1931

5. Communication personnelle des Archives Départementales concernées.

\section{RÉSUMÉS}

Située à la limite du Massif armoricain et du Bassin parisien, la forêt d'Écouves, de par sa topographie, son évolution diachronique et ses spécificités biopédologiques, s'écarte du corpus des forêts de plaines et de plateaux étudiées ces dernières années en France métropolitaine. L'étude géohistorique du massif forestier d'Écouves repose sur une démarche pluridisciplinaire s'appuyant sur des méthodes propres à l'histoire (dépouillement des archives), l'archéologie (prospection pédestre), l'archéobotanique (palynologie, datation 14C) et la géographie (cartointerprétation, intégration des informations sous SIG). Cet article propose un premier schéma de l'évolution du paysage forestier d'Écouves sur les cinq derniers siècles illustrant la construction de l'espace (dynamiques sylvicoles, transmission des formes) sur la longue durée. Si les traces d'anthropisation semblent plus ténues qu'ailleurs, ce travail souligne néanmoins la nécessité d'appréhender l'espace forestier comme un palimpseste spatial conservant des héritages anthropiques surimposés et des dynamiques biogéographiques qu'il convient de révéler.

Situated at the limits of the Armorican Massif and the Paris Basin, the Écouves Forest, due to its topography, its diachronic evolution and its special bio-pedological characteristics, differs from the corpus of forests found on plains and plateaus studied in recent years in metropolitan France. The geohistorical study of the Écouves Forest Massif is based on a multidisciplinary approach using methods employed in the fields of history (archive analysis), archaeology (prospecting on foot), archaeobotany (palynology, carbon-14 dating) and geography (the interpretation of maps and the integration of information generated by GISs). This article proposes an initial outline of the evolution of the Écouves Forest landscape over the last five centuries illustrating the construction of space (silvicultural dynamics, the transmission of forms) over a long period. Although the traces of anthropization appear to be more tenuous than elsewhere, this work stresses the need to understand the forest as a spatial palimpsest preserving superimposed anthropic legacies and bio-geographic dynamics which are of scientific interest. 


\section{INDEX}

Mots-clés : forêt, géohistoire, archéologie, parcellaires anciens, sylvigénèse, archives, biogéographie

Keywords : forest, geohistory, archaeology, ancient parcels, sylvigenesis, archives, biogeography

\section{AUTEURS}

\section{NICOLAS BLANCHARD}

Nicolas Blanchard est doctorant en géographie au sein du laboratoire IDEES (UMR 6266). Ses domaines de recherches sont la géohistoire, l'archéologie, les forêts, l'évolution des paysages, les interactions sociétés-environnements, le SIG historique.

nicolas.blanchard3[at]univ-rouen[dot]fr

\section{DAMASE MOURALIS}

Damase Mouralis est professeur en géographie à l'université de Rouen-Normandie et au laboratoire Idées (UMR 6266). Géomorphologue et géoarchéologue, il s’intéresse aux interactions sociétés-environnements, à l'évolution sur le temps long des paysages et à l'utilisation des archéo-matériaux.

damase.mouralis[at]univ-rouen[dot]fr

http://umr-idees.fr/user/damase-mouralis/

\section{DOMINIQUE TODISCO}

Dominique Todisco est maître de conférences en géographie à l'université de Rouen-Normandie au laboratoire Idées (UMR 6266). Géoarchéologue, il s'intéresse aux interactions sociétésenvironnements, à l'évolution sur le temps long des paysages et aux processus de formation des sites archéologiques.

Dominique.todisco[at]univ-rouen[dot]fr 